\title{
An improvement to hybrid beamforming precoding scheme for mmWave massive MIMO systems based on channel matrix
}

\author{
Zahra Amirifar, Jamshid Abouei \\ Department of Electrical Engineering, Yazd University, Yazd, Iran
}

\begin{tabular}{l} 
Article Info \\
\hline Article history: \\
Received Mar 24, 202 \\
Revised Jun 10, 2021 \\
Accepted Jun 18, 2021 \\
\hline Keywords: \\
5G network \\
Channel matrix \\
Hybrid beamforming \\
Massive MIMO \\
Optimization \\
Precoding
\end{tabular}

Article Info

Article history:

Received Mar 24, 2021

Revised Jun 10, 2021

Accepted Jun 18, 2021

Precoding

\begin{abstract}
The massive multiple-input multiple-output (MIMO) technology has been applied in new generation wireless systems due to growing demand for reliability and high data rate. Hybrid beamforming architectures in both receiver and transmitter, including analog and digital precoders, play a significant role in $5 \mathrm{G}$ communication networks and have recently attracted a lot of attention. In this paper, we propose a simple and effective beamforming precoder approach for mmWave massive MIMO systems. We first solve an optimization problem by a simplification subject, and in the second step, we use the covariance channel matrix $f C_{k}=\operatorname{Cov}\left(H_{k}\right)$ and $B_{k}=H_{k} H_{k}^{H}$ instead of channel matrix $H_{k}$. Simulation results verify that the proposed scheme can enjoy a higher sum rate and energy efficiency than previous methods such as spatially sparse method, analog method, and conventional hybrid method even with inaccurate Channel State Information (CSI). Percentage difference of the achievable rate of $C_{k}=\operatorname{Cov}\left(H_{k}\right)$ and $B_{k}=H_{k} H_{k}^{H}$ schemes compared to conventional methods are $2.51 \%$ and $48.94 \%$, respectively.
\end{abstract}

This is an open access article under the CC BY-SA license.

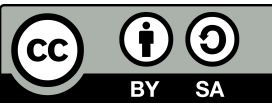

\section{Corresponding Author:}

Jamshid Abouei

Department of Electrical Engineering

Yazd University

Safaie, Yazd, Iran

Email: abouei@yazd.ac.ir

\section{INTRODUCTION}

An increase in the number of antenna arrays has been recognized as the most important factor in wireless communication due to achieving higher spectral and energy efficiencies, higher data rates, and the capability of interference mitigation [1]. For such a technology, known as massive multiple-input multipleoutput (MIMO) [2]-[4], many number of antennas can be leveraged in different structures. Among the most popular approaches, spatial multiplexing, spatial diversity, and beamforming are promising candidates for the next generation of wireless communications [5]. These technologies are certain to play a significant role in increasing the network's throughput. In recent years, beamforming algorithms have been widely used in various MIMO communications applications, in particular in mmWave systems and 5G cellular networks [6]. This technique significantly increases the array gain, provides additional radio link margin that reduces propagation path loss, and mitigates the co-channel interference. Beamforming in mmWave systems offers enormous potentials where highly directional adaptive antennas can be built in very small form factors to transmit signals for maximal signal-to-noise ratio (SNR). Despite its astounding advantages, other issues such as cost, power consumption, complexity in the corresponding optimization algorithms, channel estimation, and modeling pose 
significant challenges to the beamforming technique in massive MIMO systems [7], [8]. In an analog beamformer, the antenna array is connected to a single radio frequency (RF) chain including amplifier and analog to digital converter (ADC). Besides, its phase shifters are cheaper and have a lower power consumption compared to RF chains. Digital beamformer, on the other hand, is the optimal scheme based on the singular value decomposition (SVD) of the channel matrix $\mathrm{H}$, where a single phase array antenna is connected to a single RF chain. The optimal precoder and combiner in transmitter/receiver sides are set according to the right and left singular vectors, respectively. However, this scheme is not feasible in energy-constrained massive MIMO networks. There are some specific challenges in deploying analog and digital beamforming techniques in massive MIMO systems such as theoretical analysis with practical constraints, power consumption of RF chains, pilot contamination in the uplink, channel estimation, efficient channel feedback mechanism, and employing low-complexity signal detection algorithms [9]. However, massive MIMO systems contain a large number of antennas, hence, one should avoid using one RF chain at each antenna due to the cost, power composition, and complexity involved. On one hand, an essential option in achieving the aforementioned benefits is to employ massive MIMO technique. On the other hand, in the traditional MIMO architecture, each antenna requires to be connected to one RF chain. Hence, the fully digital beamforming solution will lead to high complexity and more energy consumption in massive MIMO scenarios, especially in mmWave frequency band [10], [11]. To solve this problem, various hybrid analog and digital beamforming structures have been proposed in the literature [12]-[15]. A key factor involved with such a hybrid beamforming is the design of small-size digital signal processor in baseband and large-size analog phase shifters in RF band to increase the antenna array gain. Therefore, hybrid beamforming can enjoy a lower number of RF chains and higher energy efficiency rather than full digital beamforming without obvious performance loss [16]. There are two hybrid beamforming architectures namely fully-connected and sub-connected structures, where each RF chain is connected to either all antennas or a set of selected antennas, respectively. The fully-connected hybrid beamforming architecture is not practical, since it requires a huge number of phase shifters which increase the computational complexity, cost, and the energy consumption [17]. In contrast, the sub-connected hybrid beamforming architecture displays a significant reduction in the number of phase shifters.

Another challenge that should be addressed for the analysis and evaluation of hybrid beamforming with sub-connected architecture is the availability of the channel state information (CSI) in transmitter and/or receiver. In the MIMO system, the CSI is gathered in the form of a matrix which is known as channel matrix $\mathrm{H}$. From the information theoretic points of view, matrix $\mathrm{H}$ has been recognized as an integral part of MIMO systems, because any channel estimation method (e.g., minimum mean square error (MMSE)), and precoding and postcoding matrices are optimized based on this matrix. The full CSI, when used appropriately achieves the highest performance of any MIMO system. However, a MIMO system is more preferable that keeps its performance and be non-sensitive under the imperfect estimation of channel matrix $\mathrm{H}$ [19]. Note that in practical situations, perfect CSI at the transmitter is not perfectly available because of feedback error, channel estimation error, the number of channel parameters, and time varying channel, while the high capacity rises with the number of paths in the channel [20]. To tackle this problem, one should employ the MIMO channel covariance matrix estimation which evaluates how much the variables change together. In this regard, we propose an effective optimization solution for sub-connected hybrid beamforming in mmWave massive MIMO systems based on the covariance of channel matrix $C_{k}=\operatorname{Cov}\left(H_{k}\right)$ and $B_{k}=H_{k} H_{k}^{H}$ instead of the channel matrix $H_{k}$.

The proposed beamforming scheme displays a higher data rate, simpler, and achieves the best possible energy efficiency. Simulation results show that our proposed structure achieves an even higher data rate when the number of received antennas is doubled. Moreover, we compute the energy efficiency of the various precoding schemes. In addition, we apply the impact of imperfect CSI on the massive MIMO system performance to show that our proposed method has a special advance which is non-sensitive to imperfect CSI.

The rest of the paper is organized as follows: describes the system model including channel matrix, the received signal vector, the achievable rates, and optimization problem in section 2 and section 3 . specifies the proposed schemes and describes well-known precoders such as spatially sparse and analog methods. The simulation results, in terms of the energy efficiency of the proposed scenario compared with the aforementioned precoders are provided in section 4. Finally, we conclude the paper in section 5.

\section{THE PROPOSED METHOD}

In this work, we consider the hybrid beamforming with sub-connected architecture the sase station (BS) is equipped with $M_{T}$ antennas connected to $N_{T} \mathrm{RF}$ chains to serve $K$ users, where $N_{T}<M_{T}$. In the 
hybrid beamforming scenario, the signal is then processed digitally by digital precoder in baseband and is then processed by analog precoder in RF domain. Accordingly, in the receiver, the received signal is processed by analog combiner and then modified by digital combiner. Finally, the received vector at user $k$ is given by [10].

$$
y_{k}=W_{B B}^{(k) H} W_{R F}^{(k) H} H_{k} F_{R F} F_{B B}^{(k)} s_{k}+W_{B B}^{(k) H} W_{R F}^{(k) H} n_{k}
$$

Where $s_{k}$ is the symbol vector of $r_{k}$ data streams with the total number of data streams $r=\sum_{k=1}^{K} r_{k}$, $W_{B B}^{(k)} \in \mathbb{C}^{N_{R_{k}} \times r_{k}}$ and $W_{R F}^{(k)} \in \mathbb{C}^{M_{R_{k}} \times N_{R_{k}}}$ denote the baseband decoder and the RF combiner, respectively. The number of receive antennas and the number of antennas connected to one RF chain are denoted by $M_{R_{k}}$ and $N_{R_{k}}$, respectively. In addition, $H_{k} \in \mathbb{C}^{M_{R_{k}} \times M_{T}}$ is the channel matrix between BS and user $k$. Similarly, $F_{R F} \in$ $\mathbb{C}^{M_{T} \times N_{T}}$ and $F_{B B}^{(k)} \in \mathbb{C}^{N_{T} \times r}$ represent the analog RF and digital baseband precoders, respectively. Finally, $n_{k} \sim$ $\mathscr{N}\left(0, \sigma^{2} I_{M_{R_{k}}}\right)$ is complex additive white gaussian noise (AWGN) vector with the independent and identical distribution (i.i.d) with zero mean and variance $\sigma^{2}$.

In this paper, we adopt the same mmWave channel model as in $[10,11,14,15]$, where it is assumed that the channel matrix is sum of the scatters in mmWave propagation environment for narrowband downlink MIMO system, i.e.,

$$
H=\beta \sum_{l=1}^{L} \alpha_{l} \Lambda_{l}\left(\varphi_{l}^{r}\right) \Lambda_{l}\left(\varphi_{l}^{t}\right) a_{r}\left(\varphi_{l}^{r}\right) a_{t}^{H}\left(\varphi_{l}^{t}\right)
$$

Where $\beta$ is a normalization factor, $L$ is the number of multipath, $\alpha_{l}$ is the gain of $l^{\text {th }}$ path, $\Lambda_{l}\left(\varphi_{l}^{t}\right)$ and $\Lambda_{l}\left(\varphi_{l}^{r}\right)$ denote the transmit and receive antenna array gains [17], $a_{r}$ and $a_{t}$ represent the array response vectors. In addition, $\varphi^{t}$ and $\varphi^{r}$ are angle of departure (AoD) and angle of arrival (AoA), respectively. Assuming that the BS and each user are equipped with linear array (LA), the array response vector with $N$ elements can be expressed as [21]:

$$
\mathbf{a}_{L A}(\varphi)=\frac{1}{N}\left[1, e^{j \frac{2 \pi}{\lambda} d \sin \varphi}, \ldots, e^{j(N-1) \frac{2 \pi}{\lambda} d \sin \varphi}\right]^{T}
$$

where $\lambda$ is the wavelength and $d$ is the antenna spacing. Additionally, mmWave channel $H$ follows the sparsescattering model because of the limited number of scatters in the environment [1].

The performance of our proposed scheme is evaluated in terms of the achievable rate of user $k$ and is compared that with other precoding methods. Hence, $R_{k}$ can be formulated as [11]:

$$
R_{k}=\log _{2}\left(\left|I_{M_{R_{k}}}+\frac{\rho}{N_{T} \sigma^{2}} H_{k} F_{R F} F_{B B}^{(k)} F_{B B}^{(k) H} F_{R F}^{H} H_{k}^{H}\right|\right)
$$

where $\rho$ denotes the transmitter power budget. In addition, we present the energy efficiency of the aforementioned schemes defined as [22]:

$$
\gamma=\frac{R_{k}}{P_{\text {total }}}=\frac{R_{k}}{P_{t}+N_{T} P_{R F}+N_{P S} P_{P S}}(\mathrm{bps} / \mathrm{Hz} / \mathrm{W})
$$

where $P_{\text {total }}$ is the total energy consumption, $N_{P S}$ is the number of required phase shifters, $P_{t}, P_{R F}, P_{P S}$ are the transmitted energy, energy consumed by RF chains and the energy consumed by phase shifters, respectively.

\section{RESEARCH METHOD}

In this section, we propose a simple and effective approach which achieves better performance than traditional precoders. Therefore, we aim to maximize the objective function in massive MIMO systems, which can be expressed as [23]:

$$
\begin{aligned}
\max _{F_{R F}, W_{R F}^{(k)}, \forall k} & \sum_{k=1}^{K}\left\|W_{R F}^{(k) H} H_{k} F_{R F}\right\|_{F}^{2} \\
\text { s.t. } & F_{R F}^{H} F_{R F}=I_{N_{T}}, W_{R F}^{(k) H} W_{R F}^{(k)}=I_{N_{R_{k}}}, \forall k
\end{aligned}
$$

Using the Frobenius norm [25], the objective function in (6) can be rewritten as:

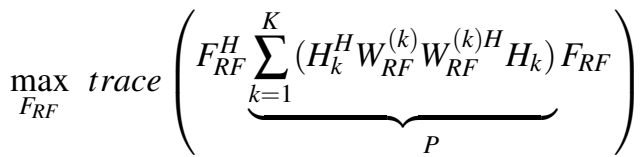

$$
\begin{aligned}
& \text { s.t. } F_{R F}^{H} F_{R F}=I_{N_{T}}
\end{aligned}
$$


for given $W_{R F}^{(k)}, \forall k$, and

$$
\begin{aligned}
& \max _{W_{R F}^{(k)}, \forall k} \operatorname{trace}(\sum_{k=1}^{K} W_{R F}^{(k) H} \underbrace{\left(H_{k} F_{R F} F_{R F}^{H} H_{k}^{H}\right)}_{Q_{k}} W_{R F}^{(k)}) \\
& \text { s.t. } W_{R F}^{(k) H} W_{R F}^{(k)}=I_{N_{R_{k}}}, \forall k
\end{aligned}
$$

for given $F_{R F}$.

The solutions of objective functions in (7) and (8) are the same. Liked by the generalized low rank approximation of matrices (GLRAM) algorithm in [23], these equations are solved by an iterative procedure. In this paper, we propose a simpler solution where we first simplify (7) and (8) and then apply the SVD method [23] on the channel matrix to compute optimal $F_{R F}$ and $W_{R F}^{(k)}$. In the proposed algorithm, the optimal precoder and combiner are set according to the right and left singular vectors, respectively. The SVD of matrix $H_{k}$ with rank $r$ can be expressed as [24]:

$$
H_{k}=U_{k} \Sigma \mathbf{V}_{k}^{H}
$$

where $U_{k}$ and $V_{k}$ are $M_{R_{k}} \times r$ and $M_{T} \times r$ dimensional unitary matrices (i.e., $U_{k}^{H} U_{k}=I_{r}$ and $V_{k}^{H} V_{k}=I_{r}$ ), respectively, and $\Sigma$ denotes $r \times r$ diagonal matrix with $\delta_{1}, \ldots, \delta_{r}$ eigenvalues. Thus, the optimal precoding matrix can be expressed as [4]:

$$
W_{R F, o p t}^{(H)}=V_{k}
$$

As a result, the matrix $H_{k}$ can be rewritten as:

$$
H_{k}=\sum_{i=1}^{r} \delta_{i} u_{i, k} v_{i, k}^{H}
$$

where $u_{i, k}$ and $v_{i, k}$ are the column vectors of matrices $U_{k}$ and $V_{k}$, respectively. In addition, the matrix $B_{k}=H_{k} H_{k}^{H}$ with dimension $M_{R_{k}} \times M_{R_{k}}$ can be decomposed as:

$$
H_{k} H_{k}^{H}=D_{k} \Lambda D_{k}^{H}
$$

where $D_{k}$ is $M_{R_{k}} \times M_{R_{k}}$ dimensional modal matrices $\left(D_{k}^{H} D_{k}=I_{M_{R_{k}}}\right)$, and $\Lambda$ denotes $\mathrm{M}_{R_{k}} \times M_{R_{k}}$ diagonal matrix with $\eta_{1}, \ldots, \eta_{M_{R_{k}}}$ eigenvalues. As a result, it is easy to show that

$$
\eta_{i}= \begin{cases}\delta_{i}^{2} & i=1,2, \ldots, r \\ 0 & i=r+1, \ldots, M_{R_{k}}\end{cases}
$$

Note that for quality of service (QoS) reasons, the number of RF chains $\left(N_{T}\right)$ ought not to be too small during the peak time when compared with the number of antennas $\left(M_{T}\right)$. Therefore, for the objective functions (7) and (8), we have $W_{R F}^{(k)} W_{R F}^{(k) H} \simeq \frac{N_{T}}{M_{T}} I_{M_{R_{k}}}$ and $F_{R F}^{(k)} F_{R F}^{(k) H} \simeq \frac{N_{T}}{M_{T}} I_{M_{T}}$, respectively. Hence, without any loss of generality, we choose these matrix substitution approaches for simplicity of problem. Therefore, problems (7) and (8) can be rewritten as:

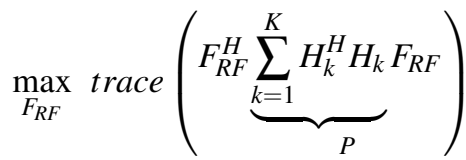

$$
\begin{aligned}
& \text { s.t. } F_{R F}^{H} F_{R F}=I_{N_{T}}
\end{aligned}
$$

and

$$
\begin{aligned}
& \max _{W_{R F}^{(k)}, \forall k} \operatorname{trace}(\sum_{k=1}^{K} W_{R F}^{(k) H} \underbrace{\left(H_{k} H_{k}^{H}\right)}_{Q_{k}} W_{R F}^{(k)}) \\
& \text { s.t. } W_{R F}^{(k) H}\left\{W_{R F}^{(k)}=I_{N_{R_{k}}}, \forall k\right.
\end{aligned}
$$

In the second step, problems (14) and (15) can be solved via the SVD method to find $F_{R F}$ and $W_{R F}^{(k)}$ including the $N_{T}$ largest eigenvectors of $P$ and the $N_{R_{k}}$ largest eigenvalues of $Q_{k}$, respectively. 
Recalling the fact that the transmitter of user $k$ sends pilot waveforms to estimate the channel responses matrix $H_{k}$, in the proposed precoder scheme for the underlying mmWave massive MIMO system, we calculate the covariance of this channel matrix, denoted by $C_{k}$, and use this matrix instead of $H_{k}$ in all precoder methods. Simulation results in Section 4. verify that our proposed structure can achieve higher rate and energy-efficiency when compared with the case when only matrix $H_{k}$ is used.

In practice, a channel matrix $H_{k}$ is described as (2). Hence, the covariance of such a matrix is calculated using the following formula

$$
C_{k}=\operatorname{Cov}\left(H_{k}\right)=E\left[\left(H_{k}-E\left(H_{k}\right)\right)\left(H_{k}-E\left(H_{k}\right)\right)^{T}\right]
$$

Thus, the formula (4) can be written as

$$
\hat{R}_{k}=\log _{2}\left(\left|I_{M_{R_{k}}}+\frac{\rho}{N_{T} \sigma^{2}} B_{k} F_{R F} F_{B B}^{(k)} F_{B B}^{(k) H} F_{R F}^{H} B_{k}\right|\right)
$$

and,

$$
\tilde{R}_{k}=\log _{2}\left(\left|I_{M_{T}}+\frac{\rho}{N_{T} \sigma^{2}} C_{k} F_{R F} F_{B B}^{(k)} F_{B B}^{(k) H} F_{R F}^{H} C_{k}\right|\right)
$$

In this paper, we compare our proposed algorithm with the conventional structure based on analog precoder, conventional hybrid precoder, and spatially sparse precoder [11].

\section{RESULTS AND DISCUSSION}

In this section, we provide some simulation results in terms of the achievable rate and the energy efficiency. The performance evaluation of the proposed precoding is also examined in detail and compared that with various well-known recently works such as analog precoding, spatially sparse precoding, and hybrid precoding. Furthermore, we assume that both transmit and receive antennas correspond to a typical uniform linear array configuration with spacing $d=\lambda / 2$. According to the rayleigh fading channel model, the aforementioned precoding schemes can be applied to the mmWave system with a few number of effective multipath components. Hence, for the channel model in (2), we define $L=3$ [9], and also carrier frequency $28 \mathrm{GHz}$ [12], AoDs and AoAs uniform distribution within the interval $\left[-\frac{\pi}{6}, \frac{\pi}{6}\right]$ and $[-\pi, \pi]$, respectively, because of using omnidirectional antennas by users. In addition, we consider SNR equal to $\rho / \sigma^{2}, M_{R_{k}}=8,16, M_{T}=64, N_{T}=16$ (number of RF chains) and $N_{R_{k}}=M_{T} / N_{T}$.

In this paper, we set $P_{R F}=250 \mathrm{~mW}$ [18], $P_{P S}=1 \mathrm{~mW}$ [12], and $P_{t}=1 \mathrm{~W}$ [26]. In the first step of our simulation, we consider the perfect CSI conditions, where the derived achievable rate expressions in (4), (17), and (18) versus SNR are simulated in Figure 1 for all precoding schemes including our proposed algorithm and for $M_{R_{k}}=8, M_{T}=64, N_{T}=16$, and $L=3$. It is seen that the proposed precoding and analog precoding have the highest and the lowest achievable rate, respectively. After that, spatially sparse precoding and hybrid precoding achieve higher rates. When we apply matrices $B_{k}$ and $C_{k}$, all precoders with using these matrices display more achievable rate compared to the case of using matrix $H_{k}$. Note that spatially sparse precoding is a strong method and has higher achievable rate than hybrid and analog schemes, since its channel matrix includes the limited paths which is suitable for sparse environments. However, as shown in Figure 1, the spatially sparse precoder has less achievable rate than our proposed precoding. Using the fact that the accuracy of CSI is affected by the number of elements of channel matrix and in spite of spatially sparse precoding, we increase the elements of channel matrix mathematically, and without adding any antennas or RF chains. To show the superiority of our proposed scheme in increasing the achievable rate, let us consider the precoders in Table 1. For instance, for hybrid precoder case, the achievable rates are 25.21, 26.22, and 43.03 in "hybrid precoding", "hybrid $C_{k}$ precoding", and "hybrid $B_{k}$ precoding" legends, respectively, where the percentage differences are $3.92 \%$ and $52.22 \%$ compared to "hybrid precoding". 
Table 1. Comparison achievable rate of all precoders at SNR=10 $d B$

\begin{tabular}{lcr}
\hline Precoding Schemes & Achievable rate & Percentage Difference \\
\hline proposed method & 27.44 & $0 \%$ \\
analog & 16.98 & $0 \%$ \\
spatially sparse & 26.69 & $0 \%$ \\
hybrid & 25.21 & $0 \%$ \\
proposed $_{B_{k}}$ method & 45.22 & $48.94 \%$ \\
analog $_{B_{k}}$ & 34.7 & $68.57 \%$ \\
spatially $_{\text {sparse }} B_{k}$ & 44.48 & $49.99 \%$ \\
hybrid $_{B_{k}}$ & 43.03 & $52.22 \%$ \\
proposed $_{C_{k}}$ method & 28.14 & $2.51 \%$ \\
analog $_{C_{k}}$ & 18.73 & $9.80 \%$ \\
spatially sparse $_{C_{k}}$ & 27.45 & $2.80 \%$ \\
hybrid $_{C_{k}}$ & 26.22 & $3.92 \%$ \\
\hline
\end{tabular}

To summarize the above arguments, we can claim that our proposed precoding scheme based on the $B_{k}$ matrix and the covariance matrix $C_{k}$ instead of channel matrix $H_{k}$ achieves the highest achievable rate with a slight increase in complexity. This issue will be decreased when the number of received antennas is larger (e.g,. $M_{R_{k}}=16, M_{T}=64$ ). Figure 2 illustrates this matter, where it is observed that the proposed precoding still has a higher achievable rate than other precoder schemes. The above results are achieved under the perfect CSI, while in the real communication systems, the conditions are not always perfect. We apply the impact of imperfect CSI on the massive MIMO system performance which can be modeled as [27]:

$$
\widehat{H}_{k}=\alpha H_{k}+\sqrt{1-\alpha^{2}} E
$$

where $\widehat{H}$ is the actual channel matrix, $E$ is the error matrix, $\alpha \in[0,1]$ is scalar and can be considered as the CSI accuracy, which means that lower $\alpha$ is quite poor accuracy of CSI and closer to 1 means closer perfect CSI. From Figure 3, it is observed that when $\alpha$ is 0.9 , the performance of the proposed precoding scheme measurably close to perfect CSI conditions $(\alpha=1)$. Hence, we can conclude that the proposed precoding is not sensitive to the CSI accuracy even when $\alpha$ is 0.5 .

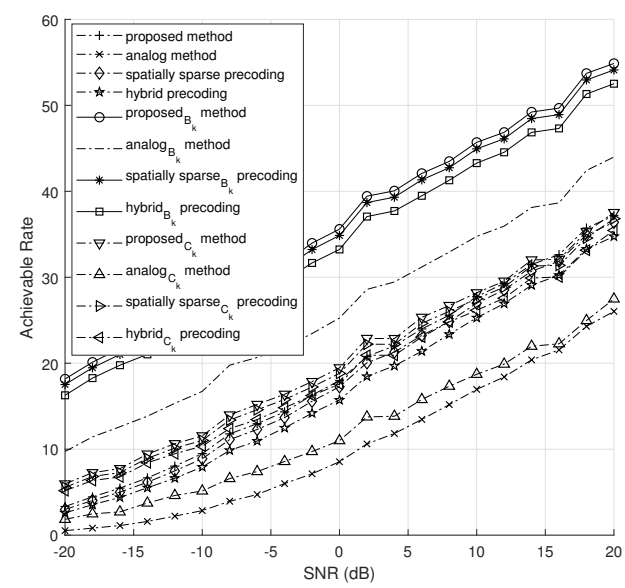

Figure 1. Achievable rate comparison versus SNR for

$M_{R_{k}}=8, M_{T}=64, N_{T}=16, L=3$

mmWave MIMO system with using $H_{k}, B_{k}$, and $C_{k}$ matrices

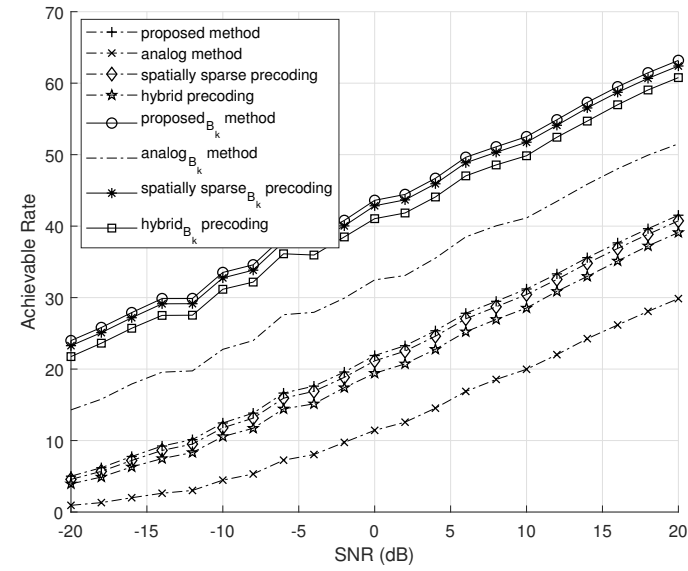

Figure 2. Achievable rate comparison versus SNR for $M_{R_{k}}=16, M_{T}=64, N_{T}=16, L=3 \mathrm{mmWave}$ MIMO system with using $H_{k}$ and $B_{k}$ matrices

The energy efficiency of the aforementioned schemes defined in (5), versus the number of RF chains $N_{T}$ is evaluated in Figure 4. We can find from Figure 4 that analog precoding based on $H_{k}$ has the lowest energy 
efficiency because of using analog elements. In addition, it is clear that our proposed precoding based on $B_{k}$ has the highest energy efficiency, especially when $N_{T}$ is lower than 30 .

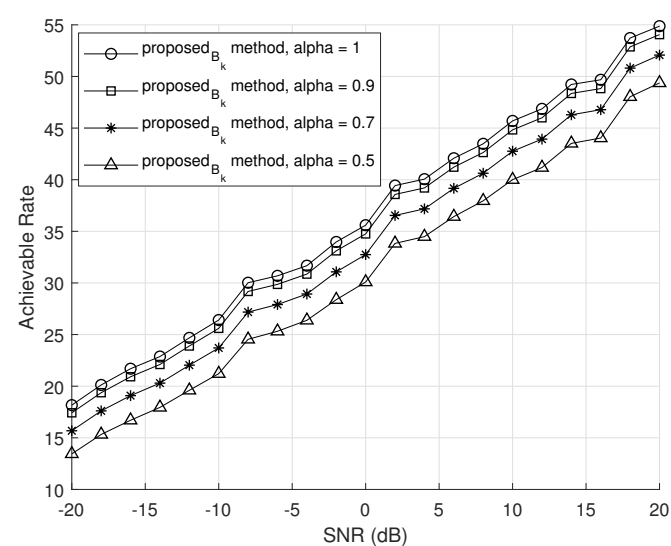

Figure 3. Impact of imperfect CSI on achievable rate comparison versus SNR for

$M_{R_{k}}=16, M_{T}=64, N_{T}=16, L=3$

mmWave MIMO system with using $B_{k}$ matrix

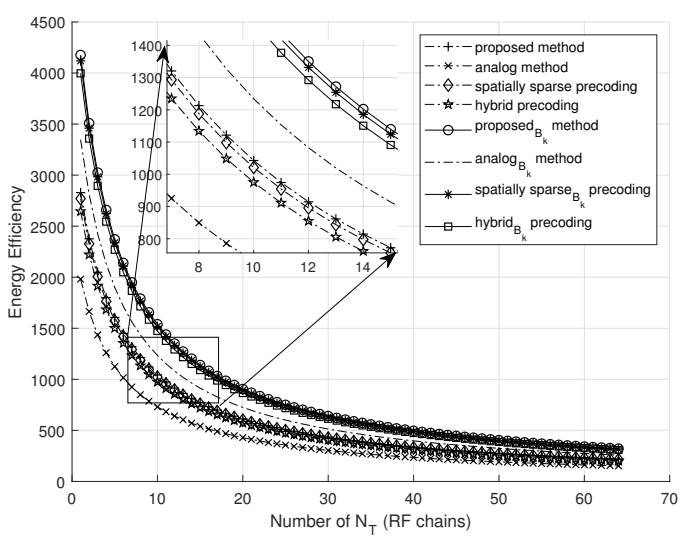

Figure 4. Energy efficiency comparison for

$$
M_{R_{k}}=8, M_{T}=64, N_{T}=16, L=3
$$

mmWave MIMO system using $H_{k}$ and $B_{k}$ matrices

\section{CONCLUSION}

This paper presented an improvement to hybrid beamforming precoding scheme suitable for mmWave massive MIMO system based on the matrices $C_{k}=\operatorname{Cov}\left(H_{k}\right)$ and $B_{k}=H_{k} H_{k}^{H}$ instead of the channel matrix $H_{k}$. As result, by applying a few more complexity, a higher achievable rate can be achieved, while this a bit more complexity will be decreased when $M_{T}$ is larger, because this complexity is about the dimension of the matrix. Our proposed precoding is also suitable when the system dimension is large. Simulation results showed that the achievable rate of the proposed precoding, spatially sparse, analog method and hybrid precoders with using $C_{k}$ and $B_{k}$ is higher than when these algorithms use only the channel matrix $H_{k}$. Furthermore, the energy efficiency of the proposed precoding was higher than other precoders. In addition, we evaluated the impact of inaccuracy of the CSI and it was shown that the proposed precoding is not sensitive to CSI accuracy. Thus, our proposed precoding has a better performance in achieving a high throughput.

\section{REFERENCES}

[1] C. G. Tsinos, S. Maleki, S. Chatzinotas, and B. Ottersten, "On the energy-efficiency of hybrid analog-digital transceivers for single- and multi-carrier large antenna array systems," IEEE Journal on Selected Areas in Communications, vol. 35, no. 9, pp. 1980-1995, Jun. 2017, doi: 10.1109/JSAC.2017.2720918.

[2] S. Hur, T. Kim, D. J. Love, J. V. Krogmeier, T. A. Thomas, and A. Ghosh, "Millimeter wave beamforming for wireless backhaul and access in small cell networks," IEEE Wireless Communications, vol. 61, no. 10, pp. 4391-4403, Sep. 2013, doi: 10.1109/TCOMM.2013.090513.120848.

[3] S. Shu, T. S. Rappaport, R. W. Heath, A. Nix, and S. Rangan, "MIMO for millimeter-wave wireless communications: Beamforming, spatial multiplexing, or both?", IEEE Communications Magazine, vol. 52, no. 12, pp. 110-121, Dec. 2014, doi: 10.1109/MCOM.2014.6979962.

[4] A. M. Sayeed and N. Behdad,“ Continuous aperture phased MIMO: Basic theory and applications," in Proc. 2010 Annual Allerton Conference on Communications, Control and Computers, pp. 1196-1203, Sep. 2010, doi: 10.1109/ALLERTON.2010.5707050.

[5] M. R. Akdeniz, Y. Liu, M. K. Samimi, S. Sun, S. Rangan, T. S. Rappaport, and E. Erkip, "Millimeter wave channel modeling and cellular capacity evaluation," IEEE J. Sel. Areas Communications, vol. 32, no. 6, pp.1164-1179, Jun. 2014, doi: 10.1109/JSAC.2014.2328154.

[6] C. Dehos, J. L. Gonzalez, A. De Domenico, D. Ktenas, and L. Dussopt, "Millimeter-wave access and backhauling: The solution to the exponential data traffic increase in 5G mobile communications systems?" IEEE Communications Magazine, vol.52, no.9, pp. 88-95, 2014, doi: 10.1109/MCOM.2014.6894457. 
[7] A. Alkhateeb, M. Jianhua, N. Gonzalez-Prelcic, and R. W. Heath Jr., "MIMO precoding and combining solutions for millimeter-wave systems," IEEE Communications Magazine, vol. 52, no.12, pp. 122-131, Dec. 2014, doi: 10.1109/MCOM.2014.6979963.

[8] X. Qing, X. Fang, M. Xiao, S. Mumtaz, and J. Rodriguez, "Beam management for millimeter-wave beamspace MU-MIMO systems," IEEE Trans. on Communications, Aug. 2018, vol. 67, no. 1, pp. 205-217, doi: 10.1109/ICCChina.2017.8330435.

[9] A. Alkhateeb, O. El Ayach, G. Leus, and R. W. Heath Jr., "Channel estimation and hybrid precoding for millimeter wave cellular systems," IEEE J. Sel. Topics Signal Process, vol. 8, no. 5, pp. 831-846, Jul. 2014, doi: 10.1109/JSTSP.2014.2334278.

[10] S. Shu, T. S. Rappaport, and M. Shaft, "Hybrid beamforming for 5G millimeter-wave multi-cell networks," IEEE Conference on Computer Communications Workshops, pp. 589-596, Apr. 2018, doi: 10.1109/INFCOMW.2018.8406871.

[11] O. El Ayach, S. Rajagopal, S. Abu-Surra, Z. Pi, and R. W. Heath Jr., "Spatially sparse precoding in millimeter wave MIMO systems," IEEE J. Sel. Areas Communications, vol. 13, no. 3, pp. 1499-1513, 2014, doi: 10.1109/TWC.2014.011714.130846.

[12] Y. Lee, C.-H. Wang, and Y.-H. Huang, "A hybrid RF/baseband precoding processor based on parallel-index-selection matrixinversion-bypass simultaneous orthogonal matching pursuit for millimeter wave MIMO systems," IEEE Trans. on Signal Processing, vol. 63, no. 2, pp. 305-317, Nov. 2014, doi: 10.1109/TSP.2014.2370947.

[13] W. Roh et al., "Millimeter-wave beamforming as an enabling technology for 5G cellular communications: Theoretical feasibility and prototype results," IEEE Communications Magazine, vol. 52, no. 2, pp. 106-113, Feb. 2014, doi: 10.1109/MCOM.2014.6736750.

[14] H. Soleimani , R. Parada, S. Tomasin , and M. Zorzi, " Fast initial access for mmWave 5G systems with hybrid beamforming using online statistics learning," IEEE Communications Magazine, vol. 57, no.9, pp. 132-7, Aug. 2019, doi: 10.1109/MCOM.2019.1800805.

[15] B. Stefano, C. DAndrea, T. Foggi, A. Ugolini, and G. Colavolpe, "Single-carrier modulation versus OFDM for millimeter-wave wireless MIMO," IEEE Trans. on Communications, vol. 66, no. 3, pp. 1335-1348, Nov. 2017, doi: 10.1109/TCOMM.2017.2771334.

[16] F. Zhu, F. Gao, M. Yao, and H. Zou, "Joint informationand jamming beamforming for physical layer security with full duplex base station," IEEE Trans. on Signal Process, vol. 62, no. 24, pp. 6391-6401, Oct. 2014, doi: 10.1109/TSP.2014.2364786.

[17] J. Juening, C. Xiao, W. Chen, and Y. Wu. "Channel-statistics-based hybrid precoding for millimeter-wave MIMO systems with dynamic subarrays," IEEE Trans. on Communications, vol. 67, no. 6, pp. 3991-4003, Feb. 2019, doi: 10.1109/TCOMM.2019.2899628.

[18] N. Khac-Hoang, S. Yang, and M. Kobayashi, "Scalable content delivery with coded caching in multi-antenna fading channels," IEEE Trans. on Wireless Communications, vol. 17, no. 1, pp. 548-562, Nov 2017, doi: 10.1109/TWC.2017.2768361.

[19] E. G. Larsson, O. Edfors, F. Tufvesson, and T. L. Marzetta, "Massive MIMO for next generation wireless systems." arXiv preprint arXiv, pp.1304.6690, Feb. 2013.

[20] J. Abouei, H. Bagheri, and A. K. Khandani, "An efficient adaptive distributed space-time coding scheme for cooperative relaying "IEEE Trans. on Wireless Communications, vol. 8, no.10, pp. 4957-4962, Oct. 2009, doi: 10.1109/TWC.2009.081615.

[21] C. A. Balanis, Antenna Theory: Analysis and Design, Wiley, USA, 2016.

[22] S. Cui, A. J. Goldsmith, and A. Bahai, "Energy-constrained modulation optimization," IEEE Trans. on Wireless Communications, vol. 4, no. 5, pp. 2349-2360, Sep. 2005, doi: 10.1109/TWC.2005.853882.

[23] D. J. Love, and R.W. Heath, "Equal gain transmission in multiple-input multiple-output wireless systems," IEEE Trans. on Communications, vol. 51 no. 7, pp. 1102-1110, Jul. 2003, doi: 10.1109/TCOMM.2003.814195.

[24] M. Hasani-Baferani, J. Abouei, and Z. Zeinalpour-Yazdi "Interference alignment in overlay cognitive radio femtocell networks," IET Communications, vol.10, no. 11, pp. 1401-1410, Jul. 2016, doi: 10.1049/iet-com.2015.0690.

[25] N. Song, T. Yang, H. Sun, "Overlapped subarray based hybrid beamforming for millimeter wave multiuser massive MIMO,” IEEE Signal Processing Letters, vol. 24, no. 5, pp. 550-554, Mar. 2017, doi: 10.1109/LSP.2017.2681689.

[26] T. S. Rappaport, J. N. Murdock, and F. Gutierrez, "State of the art in $60-\mathrm{GHz}$ integrated circuits and systems for wireless communications," Proceedings of the IEEE, vol. 99, no. 8, pp. 1390-1436, Jul. 2011, doi: 10.1109/JPROC.2011.2143650.

[27] Z. Chiya, W. Zhang, W. Wang, L. Yang, and W. Zhang, "Research challenges and opportunities of UAV millimeter-wave communications," IEEE Wireless Communications, vol. 26, no. 1, pp. 58-62, Feb. 2019, doi: 10.1109/MWC.2018.1800214. 